\title{
The Study of Fluid on Rayleigh-Taylor Instability Phenomena using Finite Volume Particle (FVP) Method
}

\author{
Nur Asiah Aprianti ${ }^{1 *}$, Sparisoma Viridi ${ }^{1}$, Zaki Su'ud ${ }^{1}$, Koji Morita ${ }^{2}$ \\ ${ }^{I}$ Physics program study ITB, Jl. Ganesha no. 10, Bandung 40132, Indonesia \\ ${ }^{2}$ Department of Applied Quantum Physics and Nuclear Engineering, Kyushu University, Moto-oka 774, Fukuoka, Japan
}

Received: 23 June 2015, Revised: 20 Aughust 2015, Accepted: 20 December 2015

\begin{abstract}
Rayleigh-Taylor instability phenomena were encountered in science world, both on a small scale and large scale (interstellar gas). Rayleigh-Taylor instability is the interpenetration of material that occurs when a fluid is above the other fluid with smaller mass density. The fluid which was originally located in the upper part will continuously broke down and the void is filled by a fluid which was originally located in lower part, so it looks like a bubble. In computational fluid dynamic field, this phenomenon is one of the benchmarks used to test the performance of buoyancy force on a numerical method. On the particle method, particularly, the Rayleigh-Taylor instability has been successfully simulated using Moving Particle Semi-implicit (MPS) method. Similar to the MPS, on this study, the Rayleigh-Taylor instability between the silicon oil and water which driven by buoyancy force was simulated using finite volume particle (FVP) method. From the simulation results it can be concluded that the shape of the bubble produced in the present calculation is similar to the results observed in the experiments and methods of MPS.
\end{abstract}

Keywords: Particle Method, Finite Volume Particle (FVP) method, Rayleigh-Taylor Instability

\section{INTRODUCTION}

In the study of fluid, Rayleigh-Taylor instability phenomenon often encountered in the interaction of two different types of fluid density. This phenomenon occurs when a fluid is above the other fluid with smaller mass density. The fluid which was originally located in the upper part will continuously broke down and the void is filled by a fluid which was originally located in lower part, so it looks like a bubble. This phenomenon has been numerically simulated by a variety of methods including particle method such as Moving Particle Semi-implicit (MPS) method by S. Zhang et. al. [3].

In the present study, the Rayleigh-Taylor instability between silicone oil and water was simulated by using the full Lagrangian method, finite volume particle (FVP), for the multi-phase flow and incompressible.

\footnotetext{
Corresponding author.

E-mail address: nura@fi.itb.ac.id
}

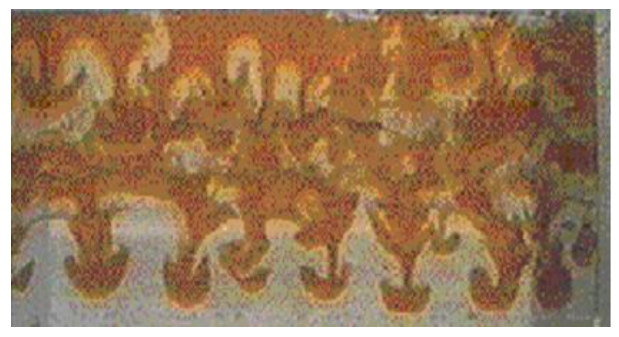

Figure 1. Rayleigh-Taylor Instability

\section{FINITE VOLUME PARTICLE METHOD}

In the FVP method, the numerical particles, which are used to discretize the governing equations, are assumed to occupy a certain volume, where the control volume of one moving particle is a circle in 2D simulations:

$$
S=2 \pi R, V=\pi R^{2}=(\Delta l)^{2}
$$

where $S, V, R$, and $\Delta l$ are the particle surface area, the particle control volume, the radius of particle control volume and the initial particle distance, respectively. According to Gauss's law, the gradient and Laplacian operators acting on 
arbitrary scalar function $\phi$ in the FVP method can be expressed by

$$
\begin{aligned}
& \nabla \phi=\lim _{R \rightarrow 0} \frac{1}{V} \oint_{V} \nabla \phi d V=\lim _{R \rightarrow 0} \frac{1}{V} \oint_{S} \phi n d S \\
& \nabla^{2} \phi=\lim _{R \rightarrow 0} \frac{1}{V} \oint_{V} \nabla^{2} \phi d V=\lim _{R \rightarrow 0} \frac{1}{V} \oint_{V} \nabla \phi \cdot{ }^{\mathrm{v}} d S
\end{aligned}
$$

where the gradient and Laplacian terms of particle $i$ can be approximated as

$$
\begin{aligned}
\langle\nabla \phi\rangle_{i} & =\left\langle\frac{1}{V} \oint_{S} \phi n d S\right\rangle_{i}=\frac{1}{V} \sum_{j \neq i} \phi_{s u r} \cdot n_{i j} \cdot \Delta S_{i j} \\
\left\langle\nabla^{2} \phi\right\rangle_{i} & =\left\langle\frac{1}{V} \oint_{S} \nabla \phi \cdot n d S\right\rangle_{i}=\frac{1}{V} \sum_{j \neq i}\left(\frac{\phi_{j}-\phi_{i}}{\left|r_{i j}\right|}\right) \cdot \Delta S_{i j}
\end{aligned}
$$

where $\langle\phi\rangle_{i}$ is the approximation of $\phi$ with respect to particle $i$ and $\left|\vec{r}_{i j}\right|$ is the distance between particles $i$ and $j$, and $\vec{n}$ is the unit vector. The function value $\phi_{\text {sur }}$ on the surface of particle $i$, can be evaluated using a linear function as

$$
\phi_{s u r}=\phi_{i}+\frac{\phi_{j}-\phi_{i}}{\left|\vec{r}_{i j}\right|} R
$$

The unit vector $\vec{n}_{i j}$ of distance between particles $i$ and $j$ is defined as

$$
\vec{n}_{i j}=\frac{\vec{r}_{i j}}{\left|\vec{r}_{i j}\right|}=\frac{\left(\vec{r}_{j}-\vec{r}_{i}\right)}{\left|\vec{r}_{i j}\right|}
$$

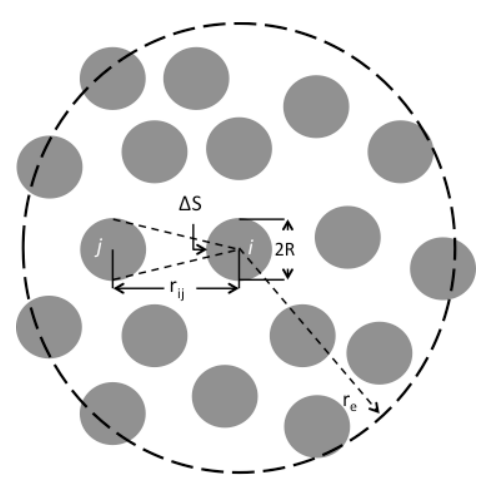

Figure 2. Neighbor particles of particle $i$ in the cut-off radius

The interaction surface $\Delta S_{i j}$ between particle $i$ and particle $j$, can be estimated as

$$
\Delta S_{i j}=\frac{\omega_{i j}}{\sum_{j \neq i} \omega_{i j}} S
$$

where $\omega_{i j}$ is the kernel function. It can be defined as

$$
\omega_{i j}= \begin{cases}\sin ^{-1}\left(\frac{R}{\mid \vec{r}_{i j}}\right)-\sin ^{-1}\left(\frac{R}{r_{e}}\right) & \left|\vec{r}_{i j}\right| \leq r_{e} \\ 0 & \left|\vec{r}_{i j}\right| \geq r_{e}\end{cases}
$$

where $r_{e}$ is the cut-off radius, which is chosen as $4.1 \Delta l$ for 2D system and $2.1 \Delta l$ for 3D system. This cut-off radius is used to define the limitation area of neighboring particles around particle $i$. Through these neighboring fluid particle interactions, the fluid movement and heat and mass transfer are modeled. The neighboring particle interactions schematically can be seen in Figure 2.

\section{GOVERNING EQUATIONS}

The governing equations to be solved for incompressible fluids are the following NavierStokes equation and the continuity equation:

$$
\begin{aligned}
& \rho \frac{D \vec{u}}{D t}=-\nabla p+\nabla(\mu \nabla \cdot \vec{u})+\vec{f}_{s}+\vec{f}_{g} \\
& \nabla \cdot \vec{u}=0
\end{aligned}
$$

where $\rho$ is the density, $\vec{u}$ is the velocity, $t$ is the time, $p$ is the pressure, $\mu$ is the dynamic viscosity, $H$ is the specific enthalpy, $k$ is the thermal conductivity and $T$ is the temperature. The third term $\vec{f}$ in the right-hand side of Eq. (10) represents other forces per unit volume, such as surface tension and gravity. The integral form of Eqs. (10) and (11), respectively, are

$$
\begin{aligned}
& \frac{D}{D t} \int_{V}^{\mathrm{V}} d V=-\frac{1}{\rho} \oint_{S} p n d S+\frac{1}{\rho} \oint_{S} \mu \nabla v \overrightarrow{\mathrm{V}} d S+\stackrel{\mathrm{V}}{f} \\
& \oint_{S}^{\mathrm{V}} \cdot \mathrm{V} d S=0
\end{aligned}
$$

By using the same approach as FVP method described in previous section, the integral form of Navier-Stokes equation, Eq. (12), can be rearranged as

$$
\frac{D u}{D t}=-\lim _{R \rightarrow 0} \frac{1}{\rho \pi R^{2}} \oint_{S} p n d S+\lim _{R \rightarrow 0} \frac{1}{\rho \pi R^{2}} \oint_{S} \mu \frac{\partial u}{\partial n} d S+\stackrel{\mathrm{V}}{f}(14)
$$

With FVP method, the Eq. (12) can be discretized as $\left(\frac{D \vec{u}}{D t}\right)_{i}=-\frac{1}{\rho \pi R^{2}} \sum_{j=1, j \neq i}\left(\mathrm{p}_{\mathrm{i}}+\frac{\mathrm{p}_{\mathrm{j}}-\mathrm{p}_{\mathrm{i}}}{\left|\overrightarrow{\mathrm{r}}_{\mathrm{ij}}\right|} \mathrm{R}\right) \Delta \bar{S}_{i j}$ $+\frac{v}{\pi R^{2}} \sum_{j=1, j \neq i} \frac{\overrightarrow{\mathrm{u}}_{\mathrm{j}}-\overrightarrow{\mathrm{u}}_{\mathrm{i}}}{\left|\overrightarrow{\mathrm{r}}_{\mathrm{ij}}\right|}\left|\Delta \bar{S}_{i j}\right|+\vec{f}$

\section{SOLUTION ALGORITHM}

\section{Pressure based solution algorithm}

There are several algorithms' solutions to solve Navier-stokes equation. In the original FVP method, PISO algorithm, which pressure based with fully implicit algorithm, is used to 
solve the problem. The disadvantage of PISO algorithm is consumed a lot of time. In the present study, for time efficiency, we adopted explicit-implicit algorithm to solve the NavierStokes equation's problem.

The flowchart of the explicit-implicit algorithm can be seen in Figure 3. In the explicit step, particle's velocity is explicitly updated with the viscosity, surface tension and external forces as

$\stackrel{\mathrm{V}}{*}^{*}=\mathrm{v}^{n}+\Delta t\left[\frac{1}{\rho} \nabla \hat{\mu} \cdot \nabla u^{n}+\frac{\hat{\mu}}{\rho} \nabla^{2} \mathrm{v}^{n}+\stackrel{\mathrm{V}}{g}+\mathrm{V}^{n}\right]$

Then particle's position is also explicitly updated as

$\stackrel{\mathrm{V}}{*}^{*}=r^{n}+\Delta t u^{*}$

In the next step, particle's velocity is updated with the solved pressure equation as

$u^{\mathrm{V}^{n+1}}=\mathrm{V}^{*}-\Delta t \frac{1}{\rho} \nabla p^{n+1}$

Then particle's position is also updated as $r^{n+1}=r^{n}+\Delta t u^{n+1}$

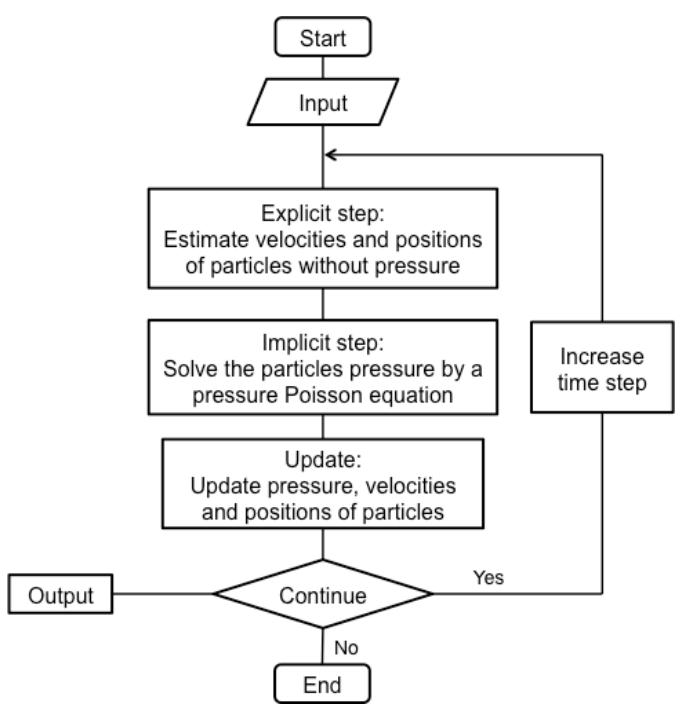

Fig. 3. Explicit-implicit algorithm.

\section{Combine-Unified Procedure (CUP) method}

To solve the pressure passion equation, a CUP method is introduced to the FVP method. The key of CUP method is linking the changes in pressure and density by introducing a sound speed and deducing an implicit expression for the pressure (Xiao et al, 1997). From the continuity equation, it can be derived as

$\frac{D \rho}{D t}=-\rho \nabla \cdot \mathrm{v}$
It can be further arranged as

$$
\nabla \cdot u=-\frac{1}{\rho} \frac{D \rho}{D t}=-\frac{1}{\rho} \frac{\partial \rho}{\partial p} \frac{D p}{D t}-\frac{1}{\rho} \frac{\partial \rho}{\partial e} \frac{D e}{D t}
$$

where $e$ is the internal energy. For the incompressible flows, assuming there are no heat conduction and dissipation, then

$$
\frac{D e}{D t}=0
$$

In addition, it is known that the sound speed can be calculated as

$$
c_{s}^{2}=\frac{\partial p}{\partial \rho}
$$

Therefore, Eq. (20) is changed into

$$
\nabla \cdot \mathrm{V}=-\frac{1}{\rho c_{s}^{2}} \frac{D p}{D t}
$$

where $c_{s}{ }^{2}$ is the smoothed square value of the sound speed. Eq. (18) can be rewritten as

$$
\nabla \cdot u^{\mathrm{V}_{n+1}}=\nabla \cdot u^{\mathrm{V}_{*}}-\nabla \cdot\left[\Delta t \frac{1}{\rho} \nabla p^{n+1}\right]
$$

According to Eq. (24), it is expressed by

$$
\nabla \cdot u^{n+1}=-\frac{1}{\rho c_{s}^{2}} \frac{p^{n+1}-p^{n}}{\Delta t}
$$

Combining Eqs. (25) and (26), we can get following equation for the pressure as

$$
-\frac{1}{\rho c_{s}^{2}} \frac{p^{n+1}-p^{n}}{\Delta t}=\nabla \cdot \cup^{*}+\frac{\Delta t}{\rho^{2}} \nabla \rho \cdot \nabla p^{n+1}-\frac{\Delta t}{\rho} \nabla^{2} p^{n+1}
$$

In order to obtain a symmetrical formulation, Eq. (27) can be changed into

$$
-\frac{1}{\rho c_{s}^{2}} \frac{p^{* *}-p^{*}}{\Delta t}=\nabla \cdot u^{\mathrm{V}_{*}}+\frac{\Delta t}{\rho^{2}} \nabla \rho \cdot \nabla p^{*}-\frac{\Delta t}{\rho} \nabla^{2} p
$$

where $p^{*}$ is initially set to be equal to $p^{n}$, and $p^{* *}$ is solved with the above equation. The value of $p^{* *}$ is then given to $p^{*}$ and Eq. (27) is solved again. As a result, iteration is generated. After convergent, $p^{* *}$ is equal to $p^{*}$, and this value is given to $p^{n+1}$ and can be solved by the Incomplete Cholesky Conjugate Gradient (ICCG) algorithm.

\section{SIMULATION CONDITIONS}

Simulation geometry of Rayleigh-Taylor instability using FVP method can be seen in figure 4. The systems consist of two fluids; water and silicon oil. Both of them placed on a $0.1 \mathrm{~m} \times 0.1 \mathrm{~m}$ 
tank. The 2 dimensions simulation is conducted with the conditions of each fluids shown in Table 1.

Table 1. Simulation conditions

\begin{tabular}{cccc}
\hline & $\begin{array}{c}\text { Mass density } \\
\left(\mathrm{Kg} / \mathrm{m}^{3}\right)\end{array}$ & $\begin{array}{c}\text { Surface } \\
\text { tension }(\mathrm{N} / \mathrm{m})\end{array}$ & $\begin{array}{c}\text { Viskosity } \\
\left(\mathrm{m}^{2} / \mathrm{s}\right)\end{array}$ \\
\hline Silicon Oil & $0.96 \times 10^{3}$ & 0.0527 & $5.0 \times 10^{-5}$ \\
Air & $1.0 \times 10^{3}$ & 0.0728 & $1.0 \times 10^{-6}$ \\
\hline
\end{tabular}

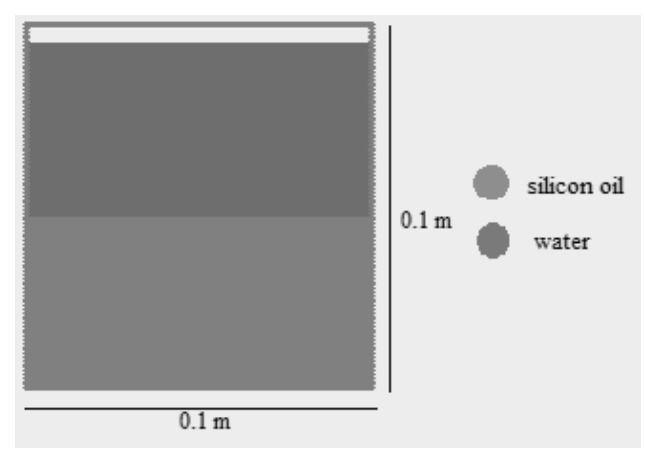

Fig. 4. Simulation geometry.

\section{RESULT AND DISCUSSIONS}

Similar to previous work"s result, MPS method, the phenomena of Rayleigh-Taylor instability can be simulate successfully using FVP method as the results shown in Table 2 .

Table 2. Simulation results

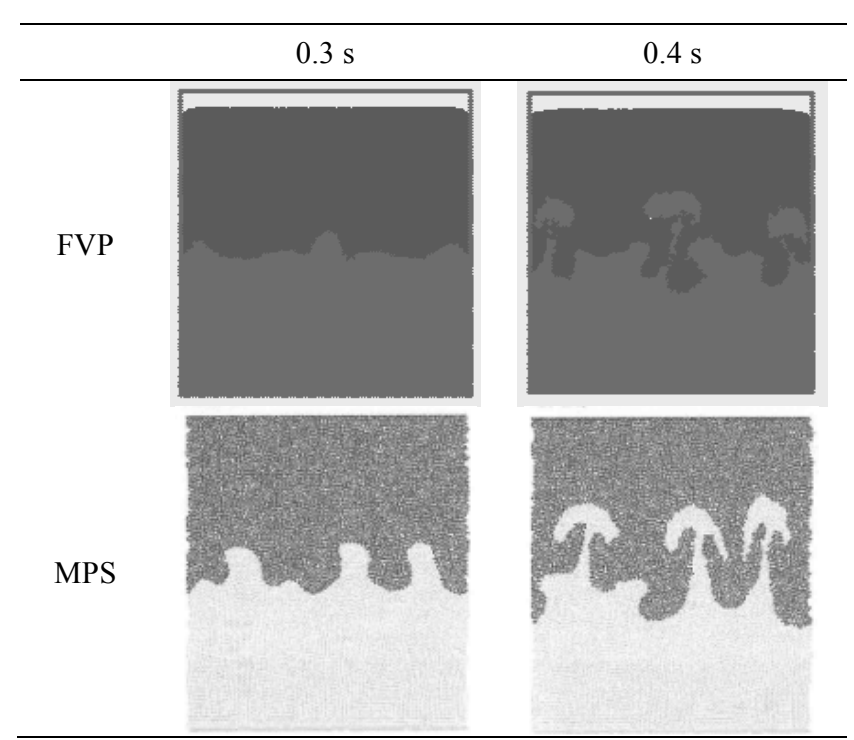

Table 2 shows the comparasion of FVP and MPS method results on Rayleigh-Taylor Instability. It can be seen that both on FVP and MPS method, the bubble as sign of Rayleigh-Taylor Instability is shaped perfectly after $0.4 \mathrm{~s}$ calculation time.

\section{CONCLUSION}

From this study, we can conclude that the Rayleigh-Taylor Instability as one of phenomena on the study of fluid can be simulate successfully using FVP method.

\section{ACKNOWLEDGMENT}

Socialication of this research is supported by research program ITB, batch II with contract number 1763/I1.B04.1/KU/2015

\section{REFERENCES}

(1) Rayleigh, Lord, Investigation of the character of the equilibrium of an incompressible heavy fluid of variable density, Proceedings of the London Mathematical Society, 14:170-177, 1883

(2) Taylor, G.I., The instability of liquid surfaces when accelerated in a direction perpendicular to their planes, Proceedings of the Royal Society of London, A201:192-196, 1950.

(3) S. Zhang, K. Morita, N. Shirakawa dan K. Fukuda; Simulation of the Rayleigh-Taylor Instability with the MPS Method, Memoirs of the faculty of Engineering, Kyushu University, Jepang, Desember 2004, Vol. 64, No. 4

(4) Nur Asiah Aprianti, Study on Particle-based Simulations of Multiphase Flows with Heat and Mass Transfer for Reactor Safety Analysis", Disertasi Doktor, Kyushu University, Jepang, 2014 